\title{
Consumo de fármacos utilizados en el tratamiento del dolor musculoesquelético en una cohorte de trabajadores manuales de Zaragoza [España]
}

\section{Drug consumption used for musculoskeletal pain treatment in a cohort of blue-collar workers in Zaragoza [Spain]}

E. Gaspar Calvo ${ }^{1}$, M. J. Lallana Álvarez y ${ }^{2}$ S. Malo ${ }^{3,4}$

${ }^{1}$ Centro de Salud Las Fuentes Norte, Servicio Aragonés de Salud. Zaragoza, España. ${ }^{2}$ Servicio de Farmacia de Atención Primaria Sector Zaragoza III, Servicio Aragonés de Salud. Zaragoza, España. ${ }^{3}$ Departamento de Medicina Preventiva y Salud Pública. Universidad de Zaragoza. ${ }^{4}$ Instituto de Investigación Sanitaria (IIS) de Aragón. Zaragoza, España

\section{ABSTRACT}

Introduction: The high prevalence of musculoskeletal diseases (MSDs) in certain populaion groups may cause a high consumption of non steroideal antiinflamatory drugs (NSAIDs). The present study aims to identify the continuous consumers of NSAIDs in a cohort of workers, who mainly perform manual tasks, analyzing the use of other drugs prescribed for the treatment of musculoskeletal pain (MSP).

Material and methods: Observational retrospective study. It has been analyzed data of all subjects, included on the AWHS (Aragon Workers Health Study) cohort, who received at least one NSAID prescription during 2016. It has been calculated the rate of use of NSAID, as well as the number of DDD per 1000 inhabitants per day (DHD). It has been identified continuous consumers, as those who used 182 defined daily dose (DDD) or more. For this group, it has been analyzed all prescriptions including other drugs used to treat MSP.

Results: The rate of use of NSAIDs in the AWHS cohort in 2016 was $41.6 \%$ (95\% Cl: 40.1\%-42.7\%), being higher in women $(47.0 \%, 95 \% \mathrm{Cl}: 41.9 \%-$ $52.1 \%$ ). $5.7 \%$ of NSAID users were continuous consumers. Among them, etoricoxib was the most used NSAIDs (4,9 DHD), followed by ibuprofen (4,0 DHD). Refering to other drugs prescribed for MSP, the prescription rate of tramadol for continuous consumers was

Gaspar Calvo E, Lallana Álvarez MJ, Malo S. Consumo de fármacos utilizados en el tratamiento del dolor musculoesquelético en una cohorte de trabajadores manuales de Zaragoza (España). Rev Soc Esp Dolor. 2020;27(3):150-159

\section{RESUMEN}

Introducción: La alta prevalencia de enfermedades musculoesqueléticas (EME) en determinados grupos de población puede condicionar que el consumo de antiinflamatorios no esteroideos (AINE) sea elevado. El presente estudio pretende identificar a los consumidores continuados de AINE en una cohorte de trabajadores que realizan fundamentalmente tareas de tipo manual, analizando el uso que hacen de otros fármacos indicados en el tratamiento del dolor musculoesquelético (DME).

Material y métodos: Estudio observacional retrospectivo, en el que se han analizado los datos, recogidos en la base de consumo farmacéutico Farmasalud, de todos los sujetos de la cohorte AWHS (Aragon Workers Health Study), que presentaron al menos una receta de un AINE durante el año 2016. Se ha calculado la tasa de utilización de AINE, así como el número de dosis diarias definidas (DDD) por 1000 habitantes por día (DHD). Se han identificado los consumidores continuados como aquellos que utilizaron 182 DDD o más. Para este grupo de consumo, se han analizado otros fármacos prescritos que suelen asociarse al tratamiento del DME.

Resultados: La tasa de utilización de AINE en la cohorte AWHS en 2016 fue del 41,6 \% (IC $95 \%$ : $40,1-42,7 \%$ ), siendo mayor en mujeres $[47,0 \%$, IC $95 \%$ : 41,9-52,1\%). El 5,7\% de los usuarios de AINE

Recibido: 18-06-2019

Aceptado: 23-03-2020

Correspondencia: Elena Gaspar Calvo egaspar@salud.aragon.es 
$24.2 \%$ (95\% Cl: 16.9\%-23.3\%) and benzodiazepines $3.0 \%$ [95\% Cl: 0.1\%-6.0\%].

Conclusions: NSAID consumption in the AWHS cohort appears to be higher than in general population. Continuous consumers preferably used NSAIDs with lower cardiovascular risk. The consumption rate of weak opioids was high for continuous users.

Key words: Non-steroidal anti-inflamatory drugs, analgesics, opioids, musculoskeletal diseases, chronic pain pharmacological therapy.

\section{INTRODUCCIÓN}

Las enfermedades musculoesqueléticas (EME) son frecuentemente referidas en la población adulta (1), constituyendo uno de los motivos de consulta médica más frecuente [2], así como la principal causa de dolor crónico [3]. La Encuesta Nacional de Salud de 2017 recoge entre los problemas de salud más prevalentes en la población española la artrosis, que afecta al 18,3 \%, y los dolores de espalda crónicos, tanto cervical $[15,8 \%$ ) como lumbar [19,8 \%] (1). Además, casi la mitad de la población refiere haber tenido dolor en las 4 últimas semanas, el cual ha interferido bastante o mucho en la vida diaria en el 9,7\% de los casos (1). Es por ello que la indicación de tratamiento analgésico es, en general, elevada.

El uso de antiinflamatorios no esteroideos (AINE) para el tratamiento de este tipo de dolencias está ampliamente extendido, tanto por su elevada prescripción cowmo por su fácil acceso como automedicación. En aquellas personas con EME cronificadas, el consumo suele ser elevado, presentando un mayor riesgo de sufrir efectos adversos debido al uso de AINE (4-11), ya sean gastrointestinales, renales o cardiovasculares.

En un estudio basado en la población finlandesa, Helin-Salmivaara y cols. encontraron un aumento del riesgo de sangrado gastrointestinal 5,2 veces (intervalo de confianza [IC] $95 \%$ : 4,7-5,9] más común entre los consumidores continuados ( $\geq 182$ DDD en un año) de AINE que en los consumidores esporádicos [12]. Por otro lado, Sánchez Serrano y cols. encontraron, en un estudio de cohortes realizado sobre población española, que el ibuprofeno y otros derivados del ácido propiónico incrementan el riesgo cardiovascular con el tiempo: riesgo relativo (IC $95 \%) 2,6(2,2-3,1)$, incremento al mes de consumo 1,0 (1,0-1,0), $p<0,001$ (13). En otro estudio de cohortes, Abajo y cols. encontraron un aumento significativo del riesgo de sufrir un infarto agudo de miocardio no fatal, asociado con el uso a largo plazo (> 1 año) de AINE $(O R=1,4 ; 1,1-1,8)$ (14). se consideraron consumidores continuados. Dentro de este grupo, el etoricoxib fue el AINE más utilizado [4,9 DHD), seguido de ibuprofeno (4,O DHD). En cuanto a otros fármacos relacionados con el tratamiento del dolor musculoesquelético, la tasa de prescripción de tramadol en los consumidores continuados de AINE fue 24,2 \% (IC $95 \%: 16,9-23,3 \%$ ) y la de benzodiazepinas 3,0 \% (IC $95 \%$ : 0,1-6,0\%).

Conclusiones: El consumo de AINE en la cohorte AWHS fue más elevado que el reportado en la población general. Los consumidores continuados utilizaron preferentemente AINE con un menor riesgo cardiovascular. La tasa de consumo de opioides débiles, en los consumidores continuados de AINE, fue elevada.

Palabras clave: Antiinflamatorios no esteroideos, analgésicos opioides, enfermedades musculoesqueléticas, tratamiento farmacológico dolor crónico.
Junto con los AINE, otros fármacos son utilizados en el manejo del dolor crónico. Analgésicos opioides y no opioides, ciertos antidepresivos, así como benzodiacepinas, se combinan con frecuencia, siguiendo las recomendaciones de la escalera analgésica de la Organización Mundial de la Salud (OMS) para el manejo del dolor, la cual fue desarrollada en 1986 (y actualizada en 1996) (15) para ayudar al control del dolor en pacientes con cáncer. En la actualidad se utiliza también en el manejo del dolor crónico, incluido el generado por las EME.

Las EME son el problema de salud relacionado con el trabajo más común en Europa. Según la Agencia Europea para la Seguridad y la Salud en el Trabajo, casi el $24 \%$ de los trabajadores de la Unión Europea afirma sufrir dolor de espalda y el $22 \%$ refiere dolores musculares, siendo el cuello, los hombros y las extremidades superiores zonas que se afectan con mayor frecuencia (16). El presente estudio se ha llevado a cabo en una cohorte compuesta fundamentalmente por trabajadores que realizan labores de producción (17), que incluyen la realización de movimientos repetitivos y el mantenimiento de posturas de forma prolongada. En este contexto se presupone una elevada prevalencia de EME, lo que determinaría un consumo elevado de AINE.

Así pues, el objetivo del presente estudio es identificar y caracterizar los diferentes grupos de consumo de AINE en una cohorte de trabajadores, con especial atención a los consumidores continuados, analizando el uso que estos hacen de otros fármacos indicados en el tratamiento del dolor.

\section{MATERIAL Y MÉTODOS}

Se ha realizado un estudio observacional retrospectivo, siendo la población de estudio todos los trabajadores de la planta de ensamblaje de vehículos Opel España en Figueruelas (Zaragoza, España), que durante 
los años 2009-2010 aceptaron participar en el Aragon Workers' Health Study (AWHS). El AWHS es un estudio de cohortes que fue diseñado para evaluar, mediante la realización de exámenes clínicos estandarizados, la trayectoria de los factores de riesgo cardiovascular tradicionales y emergentes, así como su asociación con la prevalencia y la progresión de la ateroesclerosis subclínica en una población de trabajadores de mediana edad en España [17]. La cohorte está compuesta por un total de 5650 individuos, de los cuales el 92,2 \% son hombres. El seguimiento de la cohorte continúa hoy en día. La Tabla I recoge las características basales del AWHS.

Para el presente estudio se seleccionaron los participantes del AWHS cuya prestación farmacéutica estaba cubierta por el Servicio Aragonés de Salud, que presentaron al menos una receta de un fármaco AINE durante el año 2016.

La información sobre los AINE dispensados se obtuvo del Sistema de Información de Consumo Farmacéutico de la Comunidad de Aragón (FarmaSalud), donde se incluyen todos los fármacos dispensados en las farmacias de Aragón, prescritos en receta médica oficial y con cargo al Servicio Aragonés de Salud. La identificación de los fármacos se llevó a cabo considerando el sistema de clasificación ATC (acrónimo de Anatomical, Therapeutic, Chemical) propuesto por la OMS (18). Este sistema permite identificar cada principio activo mediante un código alfanumérico de siete elementos según el grupo farmacológico, el subgrupo terapéutico, los subgrupos químicos y la sustancia química.

El Anexo I recopila los códigos ATC y los principios activos de todos los AINE utilizados en 2016 en la cohorte AWHS. Los subgrupos farmacológicos pertenecientes al grupo M01A (antiinflamatorios y antirreu- máticos no esteroideos] estudiados fueron: derivados del ácido acético (MO1AB), oxicams (MO1AC), ácido propiónico y derivados (MO1AE), combinaciones de fenamatos (MO1AG) y coxibs $(\mathrm{MO1} A \mathrm{AH})$. Se ha excluido del estudio el subgrupo MO1 AX (otros agentes antiinflamatorios y antirreumáticos no esteroideos], ya que tanto por su composición como por su mecanismo de acción no pueden ser considerados como AINE.

No se incluyó en el presente estudio el consumo a cargo de mutuas o de otras entidades aseguradoras, ni tampoco el consumo hospitalario, el procedente de recetas privadas, ni la dispensación sin receta (básicamente automedicación).

\section{Análisis de los datos}

Se realizó el análisis descriptivo de las características de los sujetos incluidos en el presente estudio, así como de las recetas dispensadas.

Para medir el consumo de AINE se calcularon las tasas de pacientes tratados con AINE, por sexo y por grupos de edad, de forma separada y conjunta. Los resultados se han expresado por cada cien sujetos. El nivel de confianza fijado para el cálculo de los IC de las tasas fue del $95 \%$. Se ha utilizado el método exacto para calcular el IC $95 \%$ de las tasas en los grupos poblacionales inferiores a 30 sujetos, mientras que para los grupos con un número igual o superior a 30 individuos se ha utilizado el método aproximado.

Para explorar la utilización de AINE en el grupo de trabajadores y clasificarlos por grupos de consumidores, se utilizó el criterio definido por Helin-Salmivaara y cols. (12), según el cual se consideraron consumidores continuados aquellos que utilizaron, al menos, 182 dosis diarias definidas (DDD) en un año; los con-

TABLA I

CARACTERÍSTICAS BASALES EN EL ARAGON WORKER'S HEALTH STUDY

\begin{tabular}{|c|c|c|}
\hline & \\
\hline & Hombres & Mujeres \\
\hline Edad (años] & $49,3 \pm 8,7$ & $40,8 \pm 11,6$ \\
\hline IMC & $27,7 \pm 3,6$ & $24,4 \pm 3,8$ \\
\hline PA sistólica $(\mathrm{mmHg})$ & $127,0 \pm 14,7$ & $111,4 \pm 13,2$ \\
\hline PA diastólica $(\mathrm{mmHg})$ & $83,8 \pm 10,1$ & $76,4 \pm 9,5$ \\
\hline Colesterol total $(\mathrm{mg} / \mathrm{dl})$ & $212,4 \pm 37,6$ & $204,3 \pm 39,9$ \\
\hline $\mathrm{cHDL}(\mathrm{mg} / \mathrm{dl})$ & $52,4 \pm 11,0$ & $66,5 \pm 14,2$ \\
\hline Triglicéridos (mg/dl] & $148,8 \pm 106,1$ & $89,7 \pm 75,5$ \\
\hline Glucosa $(\mathrm{mg} / \mathrm{dl})$ & $98,3 \pm 19,6$ & $91,2 \pm 16,3$ \\
\hline \multicolumn{3}{|l|}{ Tabaquismo } \\
\hline No fumador & $1796[35,8]$ & $146(41,8)$ \\
\hline Exfumador & $1359[27,1]$ & $46(13,2)$ \\
\hline Fumador actual & $1862[37,1]$ & $157(45,0)$ \\
\hline
\end{tabular}

cHDL: colesterol unido a lipoproteínas de alta densidad. IMC: índice de masa corporal. PA: presión arterial.

Los datos se expresan como N (\%) o media \pm desviación estándar. Reproducido con permiso de Casasnovas y cols. (14). 
sumidores entre 31 y 181 DDD se clasificaron como moderados; mientras que aquellos que presentaron una utilización igual o inferior a 30 DDD conformaron el grupo de consumidores esporádicos (12). Las DDD son unidades técnicas de medida definidas por la OMS como la dosis media, por día, para un fármaco utilizado para su indicación principal, en adultos (18).

Se estudió la distribución por sexo en los tres grupos de consumo, así como la asociación entre ambas variables mediante el test Chi cuadrado. Las diferencias de edad entre los diferentes grupos de consumo se analizaron mediante el test no paramétrico de Kruskal-Wallis.

Se ha determinado el patrón de consumo de AINE mediante el cálculo del número de DDD por 1000 habitantes por día (DHD) de los 10 principios activos más utilizados por cada grupo de consumo.

Por último, se ha estudiado en los consumidores continuados el uso de otros fármacos generalmente utilizados en el tratamiento del DME, como son los analgésicos (NO2). Dentro del subgrupo de opioides (NO2A), se ha analizado el fentanilo (NO2AB) y el tramadol (NO2AX). Respecto a otros analgésicos (NO2B), se han valorado paracetamol (NO2BEO1) y metamizol (N02BBO2). Se revisaron también las prescripciones de antidepresivos (NO6AB) y de los ansiolíticos derivados de la benzodiazepina (N05BA), concretamente el diazepan (N05BA01) y el ketazolam (N05BA10), que pueden ser utilizados como relajantes musculares según su ficha técnica.

\section{Aspectos éticos y conflicto de intereses}

Los sujetos que aceptaron participar en el AWHS firmaron un consentimiento informado (17). Todos los datos se recibieron y trabajaron de manera anónima, siendo imposible la identificación de los sujetos estudiados. Por último, el estudio en el que se enmarca este trabajo recibió la aprobación por parte del Comité Ético de Investigación Clínica de Aragón (CEICA).

La presente investigación no ha recibido ayudas específicas provenientes de agencias del sector público, sector comercial o entidades sin ánimo de lucro.

Las autoras declaran no tener conflicto de intereses.

\section{RESULTADOS}

El número total de sujetos que recibieron al menos una prescripción de un AINE en 2016 fue de 2339, de los cuales el 92,6 \% fueron hombres. El 21,3\% de los sujetos recibió dicha prescripción durante el primer trimestre de 2016. La mediana de edad de los sujetos tratados fue de 57 años (rango intercuartílico $[R l]=52-60$ ]. La Figura 1 representa la distribución de los sujetos por grupos de edad y sexo.

La tasa de utilización de AINE en la cohorte AWHS fue del 41,6 \% en 2016 (IC $95 \%$ : 40,1 \%-42,7\%), siendo mayor en mujeres [47,0 \%, IC $95 \%$ : 41,9-52,1 \%) que en hombres [41,6\%, IC $95 \%$ : 40,2-42,9\%), aunque las diferencias no fueron significativas.

Los resultados fueron similares entre los diferentes grupos de edad, tanto en hombres como en mujeres (Tabla II). Para todos los grupos de edad las tasas fueron superiores en mujeres, aunque las diferencias no fueron estadisticamente significativas.

El 53,3 \% de los usuarios de AINE fueron clasificados como consumidores esporádicos, es decir, presentaron un consumo anual menor o igual de 30 DDD. El $41 \%$ fueron clasificados como consumidores moderados (entre 31 y 181 DDD), mientras que el 5,7\% se consideraron consumidores continuados, acumulando un total de DDD anual superior a 181 (Tabla III).

El 52,8\% de los hombres realizaron un consumo esporádico frente al 5,9\% que fueron considerados consumidores continuados. En el caso de las mujeres, el 59,3\% realizó un consumo esporádico, frente al 2,9\% de consumidoras continuadas. La asociación entre el sexo y el grupo de consumo no fue significativa $(p=0,1)$.

El estudio de la edad por grupos de consumo mostró diferencias estadísticamente significativas entre los 3 grupos establecidos ( $p<0,001$ ). En los sujetos con un consumo esporádico, la mediana fue de 56 años $(R l=50-60)$, en los consumidores moderados fue de 58 años ( $R \mid=47-61$ ), mientras que en los consumidores continuados la mediana fue de 59 años $(\mathrm{RI}=56-63)$.

La utilización de AINE, medida en DHD, en los diferentes grupos de consumo se recoge en la Tabla IV, que refleja los 10 principios activos más utilizados en

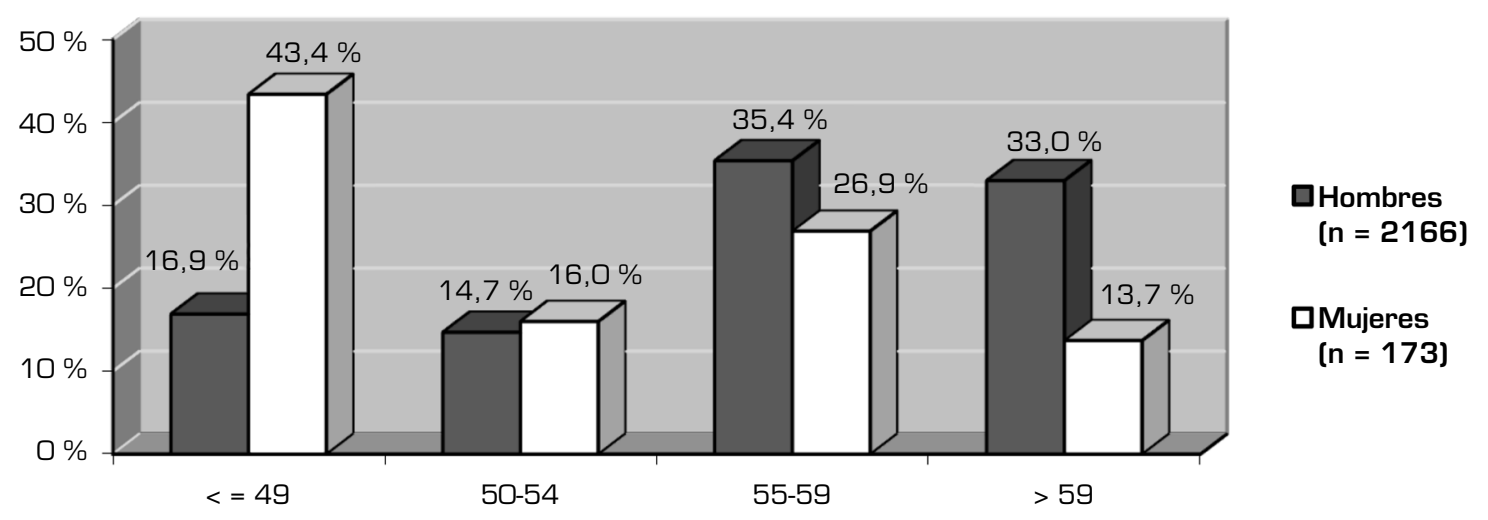

Fig. 1. Distribución por grupos de edad y sexo de los sujetos que recibieron al menos una receta de AINE. 
TABLA ॥

TASAS DE UTILIZACIÓN DE ANTIINFLAMATORIOS NO ESTEROIDEOS (AINE) EN LA COHORTE AWHS POR GRUPOS DE EDAD Y SEXO, EXPRESADAS EN TANTO POR CIEN

\begin{tabular}{|c|c|c|c|c|c|c|}
\hline Sexo & Grupos edad & $N$ & Cohorte & Tasa & ICI & ICS \\
\hline \multirow{4}{*}{ Hombres } & $\leq 49$ & 376 & 994 & $37,8 \%$ & $34,81 \%$ & $40,84 \%$ \\
\cline { 2 - 7 } & $50-54$ & 322 & 723 & $44,5 \%$ & $40,91 \%$ & $48,16 \%$ \\
\cline { 2 - 7 } & $55-59$ & 774 & 1788 & $43,3 \%$ & $40,99 \%$ & $45,59 \%$ \\
\cline { 2 - 7 } & $>59$ & 649 & 1704 & $38,1 \%$ & $35,78 \%$ & $40,39 \%$ \\
\hline \multirow{4}{*}{ Mujeres } & $\leq 49$ & 75 & 183 & $41,0 \%$ & $33,86 \%$ & $48,11 \%$ \\
\cline { 2 - 7 } & $50-54$ & 28 & 56 & $50,0 \%$ & $31,48 \%$ & $68,52 \%$ \\
\cline { 2 - 7 } & $55-59$ & 47 & 78 & $60,3 \%$ & $49,40 \%$ & $71,12 \%$ \\
\cline { 2 - 7 } & $>59$ & 23 & 51 & $45,1 \%$ & $26,67 \%$ & $63,53 \%$ \\
\hline
\end{tabular}

ICl: intervalo de confianza inferior. ICS: intervalo de confianza superior.

TABLA III

CLASIFICACIÓN DE LOS USUARIOS DE ANTIINFLAMATORIOS NO ESTEROIDEOS (AINE) SEGÚN SU NIVEL DE CONSUMO, MEDIDO A TRAVÉS DEL NÚMERO DE DOSIS DIARIAS DEFINIDAS (DDD) DISPENSADAS EN EL AÑO 2016, Y SU DISTRIBUCIÓN POR SEXO

\begin{tabular}{|c|c|c|c|c|c|c|}
\hline & \multicolumn{2}{|c|}{ Total } & \multicolumn{2}{|c|}{ Hombres } & \multicolumn{2}{|c|}{ Mujeres } \\
\hline Grupos de consumo & $N$ & $\%$ & $N$ & $\%$ & $N$ & $\%$ \\
\hline $\begin{array}{l}\text { Consumidores esporádicos } \\
{[\leq 30 D D D]}\end{array}$ & 1245 & $53,3 \%$ & 1143 & $52,8 \%$ & 102 & $59,3 \%$ \\
\hline $\begin{array}{l}\text { Consumidores moderados } \\
\text { (31-181 DDD) }\end{array}$ & 958 & $41,0 \%$ & 893 & $41,3 \%$ & 65 & $37,8 \%$ \\
\hline $\begin{array}{l}\text { Consumidores continuados } \\
{[\geq 182 \text { DDD }]}\end{array}$ & 132 & $5,7 \%$ & 127 & $5,9 \%$ & 5 & $2,9 \%$ \\
\hline Total & 2335 & $100,0 \%$ & 2163 & $100,0 \%$ & 172 & $100,0 \%$ \\
\hline
\end{tabular}

TABLA IV

NÚMERO DE DOSIS DIARIAS DEFINIDAS POR 1000 HABITANTES POR DÍA (DHD) DE LOS 10 PRINCIPIOS ACTIVOS MÁS UTILIZADOS, POR GRUPO DE CONSUMIDORES DE ANTIINFLAMATORIOS NO ESTEROIDEOS (AINE)

\begin{tabular}{|l|c|l|c|l|c|}
\hline \multicolumn{2}{|c|}{$\begin{array}{c}\text { Consumidores esporádicos } \\
{[\leq 30 D D D]} \\
n=1245\end{array}$} & \multicolumn{2}{c|}{$\begin{array}{c}\text { Consumidores moderados } \\
\text { [31-181 DDD] } \\
n=958\end{array}$} & \multicolumn{2}{c|}{$\begin{array}{c}\text { Consumidores continuados } \\
{[\geq 182 \text { DDD }} \\
n=132\end{array}$} \\
\hline \multicolumn{1}{|c|}{ Principio activo } & $D H D$ & \multicolumn{1}{|c|}{ Principio activo } & $D H D$ & \multicolumn{1}{|c|}{ Principio activo } & $D H D$ \\
\hline Ibuprofeno & 4,8 & Ibuprofeno & 11,2 & Etoricoxib & 4,9 \\
\hline Dexketoprofeno & 1,9 & Naproxeno & 5,9 & Ibuprofeno & 4,0 \\
\hline Diclofenaco & 1,9 & Diclofenaco & 4,4 & Naproxeno & 4,1 \\
\hline Aceclofenaco & 0,9 & Etoricoxib & 4,3 & Diclofenaco & 1,9 \\
\hline Celecoxib & 0,3 & Dexketoprofeno & 2,4 & Celecoxib & 1,3 \\
\hline Etoricoxib & 0,3 & Aceclofenaco & 2,0 & Dexketoprofeno & 0,8 \\
\hline Lornoxicam & 0,2 & Celecoxib & 1,4 & Aceclofenaco & 0,5 \\
\hline Meloxicam & 0,1 & Meloxicam & 0,5 & Meloxicam & 0,4 \\
\hline Naproxeno & 0,0 & Lornoxicam & 0,3 & Indometacina & 0,1 \\
\hline Dexibuprofeno & 0,0 & Indometacina & 0,1 & Tenoxicam & 0,0 \\
\hline
\end{tabular}


TABLA V

OTROS FÁRMACOS UTILIZADOS POR LOS CONSUMIDORES CONTINUADOS DE ANTIINFLAMATORIOS NO ESTEROIDEOS (AINE)

\begin{tabular}{|c|c|c|c|c|}
\hline Analgésicos & Frecuencia & Tasa & ICI & ICS \\
\hline Fentanilo & 3 & $2,3 \%$ & $-0,3 \%$ & $4,8 \%$ \\
\hline Tramadol & 32 & $24,2 \%$ & $16,9 \%$ & $23,3 \%$ \\
\hline Metamizol & 29 & $22,0 \%$ & $14,0 \%$ & $30,0 \%$ \\
\hline Paracetamol & 56 & $42,4 \%$ & $50,9 \%$ & $42,3 \%$ \\
\hline Antidepresivos & Frecuencia & Tasa & $I C I$ & ICS \\
\hline ISRS & 3 & $2,3 \%$ & $-0,3 \%$ & $4,8 \%$ \\
\hline Benzodiacepinas & 4 & $3,0 \%$ & $0,1 \%$ & $6,0 \%$ \\
\hline
\end{tabular}

ISRS: inhibidores selectivos de la recaptación de la serotonina. Benzodiacepinas: diazepam y ketazolam.

cada grupo. Los consumidores esporádicos utilizaron preferentemente ibuprofeno $[4,8 \mathrm{DHD}$ ), seguido de dexketoprofeno y diclofenaco, 1,9 DHD cada uno. Los consumidores moderados utilizaron sobre todo ibuprofeno, con 11,2 DHD. En el grupo de los consumidores continuados el etoricoxib fue el AINE más utilizado [4,9 DHD), seguido de ibuprofeno [4,O DHD).

El análisis descriptivo de otros fármacos utilizados por el grupo de consumidores continuados de AINE se recoge en la Tabla V. Con respecto al tratamiento farmacológico del DME, en el grupo de los analgésicos destaca el uso de paracetamol, cuya tasa fue del 42,4 \% (IC $95 \%$ : 50,9-42,3\%). La utilización de tramadol fue del 24,2 \% (IC $95 \%$ : 16,9-23,3\%), mientras que la tasa de utilización de benzodiazepinas entre los consumidores continuados de AINE fue del 3,0\% (IC $95 \%$ : 0,1-6,0\%).

\section{DISCUSIÓN}

Casi la mitad de los sujetos de la cohorte AWHS recibieron, al menos, una receta de AINE en 2016 y la tasa de utilización fue mayor en mujeres. Los consumidores continuados representan alrededor de un $6 \%$ de todos los usuarios de AINE y su mediana de edad fue mayor que la de los consumidores moderados y esporádicos. Además de AINE, los consumidores continuados utilizaron paracetamol, tramadol, diazepam y ketazolam.

Otros estudios han reflejado la tasa de utilización de AINE en población general (19). Un estudio llevado a cabo en Navarra revela que el $14,0 \%$ de la población fue tratada con un AINE en algún momento de los tres primeros meses del año 2016, una tasa 7 puntos más baja que la encontrada en la cohorte AWHS para el mismo periodo. Esta diferencia puede ser debida a que la mediana de edad en el subgrupo de la cohorte estudiado es elevada. La prevalencia de las EME aumenta con la edad, por lo que parece razonable que el consumo de AINE sea mayor en la cohorte que en la población general. Por otro lado, se debe tener en cuenta el hecho de que los individuos estudiados son trabajadores manuales en su mayor parte (17). La Encuesta Nacional de Condiciones de Trabajo 2015 recoge que el $84 \%$ de los trabajadores encuestados señala que está expuesto "siempre o casi siempre" o "a menudo" a algún aspecto deficiente relativo a las demandas físicas de su puesto de trabajo. Las demandas físicas más habituales son repetir los mismos movimientos de manos o brazos [69,0 \%) y adoptar posturas dolorosas o fatigantes (54,0\%) (20). Por ello, la incidencia de EME en la cohorte AWHS podría ser mayor que en la población general, lo que justificaría la elevada tasa de utilización de AINE.

Según el Informe Mujer y Salud 2008-2009 en España, es 1,8 veces más probable que las mujeres presenten algún problema crónico y 1,5 veces más probable que vean limitada su actividad a causa de ese problema, en comparación a los hombres, independientemente de su edad, la clase social, el nivel cultural o la situación laboral. En relación con las EME que padecen las mujeres, se observa que la artrosis aumenta con la edad y es más frecuente entre los 45 y 64 años. Los dolores de espalda (cervical y lumbar) también aumentan con la edad, pero alcanzan prevalencias altas ya en el grupo de 30 a 44 años [21]. Todo ello podría justificar un consumo más elevado de AINE en el sexo femenino. En nuestro estudio se ha comprobado una tasa de utilización de AINE mayor en mujeres que hombres, sin embargo, las diferencias no han sido significativas. Ello es atribuible a que el número de mujeres en la cohorte AWHS, así como entre los consumidores de AINE es bajo.

La utilización de AINE por grupos de consumo en la cohorte AWHS no sigue el patrón de prescripción por principio activo de la población española en 2016: ibuprofeno, naproxeno, etoricoxib [2]. Sorprende que este último haya sido el principio activo más prescrito entre los consumidores continuados, por delante del ibuprofeno y el naproxeno, a pesar de que estos últimos sean considerados más seguros desde el punto de vista cardiovascular [22]. Además, en este subgrupo el consumo de etoricoxib fue sensiblemente superior $(4,9 \mathrm{DHD})$ al de la población española en 2016 (3,6 DHD). 
En relación con otros fármacos utilizados por los consumidores continuados de AINE, destacamos que casi la mitad recibieron paracetamol durante el año 2016. Es uno de los medicamentos más utilizados para tratar el dolor, debido a su eficacia y su elevado perfil de seguridad. Sin embargo, una revisión sobre datos recientes señala la toxicidad, tanto gastrointestinal como cardiovascular, del paracetamol, así como su baja capacidad de analgesia en la artrosis (23), lo que ha llevado a la Osteoarthritis Research Society International (OARSI) a modificar su recomendación respecto a la utilización de este fármaco en pacientes con comorbilidades como "incierta" (24). Por ello, sería recomendable revisar y detectar, entre los sujetos de la cohorte, aquellos casos en los que la prescripción de paracetamol no sea aconsejable.

En el presente estudio, se encontró una tasa de utilización de tramadol elevada, entre los consumidores continuados de AINE de la cohorte. Este hallazgo estaría en línea con el informe del Servicio Catalán de Salud sobre los riesgos asociados al uso concomitante de opioides y benzodiacepinas, indica que, en 2016, el 6,4\% de la población catalana recibió una o más prescripciones de un fármaco opioide, de los cuales el 73,3\% utilizó tramadol (25). En el mismo sentido, diversas voces en nuestro país alertan de que el consumo de estos potentes analgésicos se ha disparado en los últimos años $(25,26)$. El informe de utilización de medicamentos opioides en España elaborado por la Agencia Española de Medicamentos y Productos Sanitarios (AEMPS) refleja el aumento del consumo del tramadol, desde 1,8 DHD en 2008 a 5,3 DHD en 2015 [27]. Por el contrario, un estudio poblacional portugués que analiza el uso de diferentes fármacos para el alivio del dolor en pacientes con dolor lumbar crónico activo, encontró un nivel de utilización de los opioides muy bajo $(1,6 \%$ ), incluso para aquellos que presentaban dolor severo [28]. El hecho de que la recogida de la información se realizara mediante entrevista personal y no a través de bases de datos de dispensación farmacéutica ha podido influir en la obtención de esas tasas tan bajas. Cabe recordar los efectos negativos de los opioides, incluido el tramadol, sobre el sistema nervioso central y el riesgo de abuso y adicción, si se usa durante más de seis meses [26]. Por ello, estos fármacos están considerados como un tratamiento de segunda línea en el manejo del dolor crónico no oncológico y, según recomiendan las guías de práctica clínica, su uso debería considerarse únicamente cuando los beneficios esperados en cuanto a dolor y función sean mayores al riesgo del paciente (Recomendación categoría A; evidencia tipo 3) [29].

Otro de los fármacos utilizados por los consumidores continuados, en relación con el tratamiento del dolor crónico por su efecto miorrelajante, fueron las benzodiacepinas. Estudios poblacionales recogen la tasa de prescripción de estos fármacos en diferentes países, observándose una amplia variabilidad. En Irlanda, la tasa en mayores de 16 años fue 16,6\% (IC 95 \%: 16,5-16,7\%) en 2015 (30). En un estudio realizado en Suiza, el 9,1\% de los adultos encuestados ( $n=45.309$ ) habían recibido al menos una prescripción en un periodo de 6 meses (31). En Estados Unidos, la encuesta nacional sobre gasto médico
Medical Expenditure Panel Survey (MEPS), realizada en 2013, indicó que el 5,6\% de los adultos mayores de 18 años habían recibido, al menos, una prescripción de benzodiacepina [32]. Sin embargo, no se han encontrado estudios en los que se cuantifique el uso de benzodiacepinas como miorrelajantes de forma explícita. Por otro lado, la prevalencia de los trastornos de ansiedad en España es del 6,2 \%, según el Estudio Europeo sobre la Epidemiología de los Trastornos Mentales (ESEMeD-España) (33); el resultado hallado en nuestro estudio podría hacernos pensar en un uso bajo de estos fármacos para el tratamiento de EME. Sin embargo, la base de datos analizada no recoge información sobre el diagnóstico asociado a la prescripción, por lo que no se ha podido comprobar la indicación terapéutica de los mismos.

\section{Limitaciones y fortalezas}

Como limitaciones del estudio, se debe tener en cuenta que la DDD es una unidad técnica de medida y que no necesariamente refleja la dosis diaria realmente prescrita o utilizada por el paciente, aunque debería aproximarse a ella. Por otro lado, los datos manejados no permiten conocer el cumplimiento de los tratamientos; por eso, los términos utilización (o consumo) no deben interpretarse en un sentido literal. En cualquier caso, se trata de un estudio de "vida real", que aporta información sobre la práctica terapéutica habitual.

Queda fuera de este estudio la automedicación y los consumos hospitalarios y privados. Sin embargo, se presupone que el consumo sin prescripción médica [OTC en inglés, over the counter] puede ser elevado, ya que otros estudios consultados así lo refieren. En Noruega una gran encuesta poblacional encontró que la prevalencia de uso de AINE OTC fue el 19,0\% (34). Otra encuesta realizada en 2009 en Australia encontró que el 26,0 \% había utilizado un AINE OTC, fundamentalmente ibuprofeno (99,0 \%) [35]. En España la Encuesta Nacional de Salud indica que el 10,1\% de la población se automedicó en 2016 y el 29,9\% de los fármacos utilizados fueron medicamentos para el dolor [36].

Para el presente estudio no se pudieron conocer datos sobre la existencia de EME previas u otras comorbilidades que podrían haber influido en el consumo de AINE de la cohorte analizada.

Las características de la cohorte, con baja presencia de mujeres y un rango de edad muy limitado, hacen que la población estudiada no sea representativa de la población general. Sin embargo, el estudio de una población activa resulta relevante, ya que es menos demandante de atención sanitaria que otros grupos poblacionales (niños y ancianos, por ejemplo) y no suele ser objeto de intervenciones sanitarias específicas.

Como fortalezas, destacamos que la base de datos analizada en el presente estudio, Farmasalud, ha sido utilizada en otros estudios previos (37-40), constituyendo una herramienta válida y con una buena calidad de registro. Del mismo modo, queremos subrayar el hecho de que no existan estudios recientes en España sobre 
la utilización de AINE y otros fármacos relacionados con el tratamiento de las EME.

\section{CONCLUSIONES}

Como conclusión, este estudio muestra la elevada utilización de AINE en una cohorte de trabajadores de tipo fundamentalmente manual. Además, los consumidores continuados utilizaron preferentemente AINE con menor riesgo cardiovascular, como etoricoxib o ibuprofeno. Por otro lado, se aportan datos sobre el uso que estos realizaron de otros fármacos que se utilizan en el manejo del dolor crónico producido por las EME, como son el paracetamol, el tramadol y las benzodiazepinas.

A tenor de los resultados obtenidos, podrian desarrollarse otras investigaciones focalizadas en grupos poblaciones con alta prevalencia de EME, como por ejemplo mayores de 65 años o mujeres, que permitieran asociar prescripción a diagnóstico médico, con el objetivo de determinar la magnitud del consumo de AINE, cuyo uso está asociado a un aumento del riesgo cardiovascular, digestivo y renal, así como de otros fármacos, como los opioides, por el conocido riesgo de abuso que existe.

\section{ANEXO 1 \\ PRODUCTOS ANTIINFLAMATORIOS Y ANTIRREUMÁTICOS NO ESTEROIDEOS UTILIZADOS POR ALGUNO DE LOS SUJETOS DE LA COHORTE AWHS}

\begin{tabular}{|c|c|c|}
\hline $\begin{array}{l}\text { M01A: Productos antiinflamatorios } \\
\text { y antirreumáticos no esteroideos }\end{array}$ & Código ATC & Principio activo \\
\hline \multirow{4}{*}{$\begin{array}{l}\text { MO1AB: derivados del ácido } \\
\text { acético y sustancias relacionadas }\end{array}$} & M01AB01 & Indometacina \\
\hline & M01AB05 & Diclofenaco \\
\hline & M01AB16 & Aceclofenaco \\
\hline & M01AB55 & Diclofenaco, asociaciones \\
\hline \multirow{4}{*}{ M01AC: oxicanes } & M01AC01 & Piroxicam \\
\hline & M01ACO2 & Tenoxicam \\
\hline & M01AC05 & Lornoxicam \\
\hline & M01ACO6 & Meloxicam \\
\hline \multirow{8}{*}{$\begin{array}{l}\text { MO1AE: derivados del ácido } \\
\text { propiónico }\end{array}$} & M01AE01 & Ibuprofeno \\
\hline & M01AEO2 & Naproxeno \\
\hline & M01AEO3 & Ketoprofeno \\
\hline & M01AE14 & Dexibuprofeno \\
\hline & M01AE17 & Dexketoprofeno \\
\hline & M01AE52 & Naproxeno y esomeprazol \\
\hline & M01AE92 & Ibuprofeno arginina \\
\hline & M01AE93 & Ibuprofeno lisina \\
\hline M01AG: fenamatos & M01AG01 & Ácido Mefenámico \\
\hline \multirow{2}{*}{ MO1AH: coxibs } & $\mathrm{MO1AHO1}$ & Celecoxib \\
\hline & M01AH05 & Etoricoxib \\
\hline
\end{tabular}

\section{BIBLIOGRAFÍA}

1. Instituto Nacional de Estadística. Encuesta Nacional de Salud, 2017 [citado el 1 de marzo de 2020]. Disponible en: https://www.ine.es/dyngs/INEbase/es/operacion.htm?c =Estadistica_C\&cid=1254736176783\&menu=resultados\& idp $=1254735573175$

2. Departamento de Medicamentos de Uso Humano de la AEMPS. Utilización de medicamentos atiinflamatorios no esteroideos en España durante el periodo 2013-2016.
Madrid: Agencia Española de Utilización de Medicamentos y productos sanitarios, Ministerio de Sanidad, Seguridad Social e lgualdad; 2017.

3. Ministerio de Sanidad servicios sociales e igualdad. Documento Marco para la mejora del abordaje del dolor en el SNS; 2014

4. Harirforoosh S, Asghar W, Jamali F. Adverse effects of nonsteroidal antiinflammatory drugs: an update of gastrointestinal, cardiovascular and renal complications. J Pharm Pharm Sci. 2013;16(5):821-47. DOI: 10.18433/j3vw2f. 
5. Arfè A, Scotti L, Varas-Lorenzo C, Nicotra F, Zambon A, Kollhorst B, et al. Non-steroidal anti-inflammatory drugs and risk of heart failure in four European countries: nested case-control study. BMJ. 2016;354:i4857. DOl: 10.1136/bmj.i4857.

6. Baigent C, Bhala N, Emberson J, Merhi A, Abramson S, Arber $\mathrm{N}$, et al. Vascular and upper gastrointestinal effects of non-steroidal anti-inflammatory drugs: Meta-analyses of individual participant data from randomised trials. Lancet. 2013;382(9894):769-79. DOI: 10.1016/S01406736(13)60900-9.

7. McGettigan P, Henry D. Cardiovascular risk and inhibition of cyclooxygenase: A systematic review of the observational studies of selective and nonselective inhibitors of cyclooxygenase 2. JAMA. 2006;296(13):1633-44. DOI: 10.1001/ jama.296.13.jrv60011.

8. Schmidt M, Lamberts M, Schjerning Olsen AM, Fosbøll E, Niessner A, Tamargo J, et al. Cardiovascular safety of non-aspirin non-steroidal anti-inflammatory drugs: review and position paper by the working group for Cardiovascular Pharmacotherapy of the European Society of Cardiology. Eur Heart J. 2016;37(13):1015-23. DOI: 10.1093/eurheartj/ehv505.

9. Schjerning Olsen AM, Gislason GH, McGettigan P, Fosbøl E, Sørensen R, Hansen ML, et al. Association of NSAID Use With Risk of Bleeding and Cardiovascular Events in Patients Receiving Antithrombotic Therapy After Myocardial Infarction. JAMA. 2015;313(8):805-14. DOI: 10.1001/ jama.2015.0809.

10. Lapi F, Azoulay L, Yin H, Nessim SJ, Suissa S. Concurrent use of diuretics, angiotensin converting enzyme inhibitors, and angiotensin receptor blockers with non-steroidal anti-inflammatory drugs and risk of acute kidney injury: nested case-control study. BMJ. 2013;346:e8525. DOI: 10.1136/bmj.e8525.

11. Moore N, Pollack C, Butkerait P. Adverse drug reactions and drug-drug interactions with over-the-counter NSAIDs. Ther Clin Risk Manag. 2015;11:1061-75. DOI: 10.2147/ TCRM.S79135.

12. Helin-Salmivaara A, Huupponen R, Virtanen A, Lammela J, Klaukka T. Frequent prescribing of drugs with potential gastrointestinal toxicity among continuous users of non-steroidal anti-inflammatory drugs. Eur J Clin Pharmacol. 2005;61(56):425-31. DOl: 10.1007/s00228-005-0949-y.

13. Sánchez Serrano JL, Tenias Burillo JM, Arias Arias A, Muñoz Carreras MI, Valenzuela Gámez JC. Riesgo cardiovascular asociado al consumo de antiinflamatorios no esteroideos. Estudio de cohortes retrospectivo en un área de salud, 2008-2012. Rev Esp Salud Publica 2015;89(6):1-7. DOI: 10.4321/S1135-57272015000600008.

14. de Abajo FJ, Gil MJ, García Poza P, Bryant V, Oliva B, Timoner $J$, et al. Risk of nonfatal acute myocardial infarction associated with non-steroidal antiinflammatory drugs, nonnarcotic analgesics and other drugs used in osteoarthritis: A nested case-control study. Pharmacoepidemiol Drug Saf. 2014;23(11):1128-38. DOI: 10.1002/pds.3617.

15. Organización Mundial de la Salud. Alivio del dolor en el cáncer. Ginebra; 1996.

16. Trastornos musculoesqueléticos - Salud y seguridad en el trabajo - EU-OSHA [Internet]. [citado 1 de marzo de 2020]. Disponible en: https://osha.europa.eu/es/themes/musculoskeletal-disorders

17. Casasnovas JA, Alcaide V, Civeira F, Guallar E, Ibañez B, Borreguero $\mathrm{JJ}$, et al. Aragon workers' health study - design and cohort description. BMC Cardiovasc Disord. 2012;12:45. DOI: 10.1186/1471-2261-12-45.
18. WHOCC - ATC/DDD Index [Internet]. Oslo; [citado 21 de febrero de 2018]. Disponible en: https://www.whocc.no/ atc_ddd_index/

19. Aranguren Ruiz I, Elizondo Rivas G AAA. Consideraciones de seguridad de los AINE. Boletín Inf Farmacoter Navarra. 2016;24(2):1-13.

20. Pinilla García J, Almodóvar Molina A, Galiana Blanco ML, Hervás River P, Zimmermann Verdejo M. Encuesta Nacional de Condiciones de Trabajo. 2015 6a EWCS - España. Madrid: Instituto Nacional de Seguridad e Higiene en el Trabajo (INSHT), Ministerio de Empleo y Seguridad Social; 2017.

21. SEMERGEN | Sociedad Española de Médicos de Atención Primaria [Internet]. [citado 28 de junio de 2018]. Disponible en: https://www.semergen.es/index. php?seccion=dinamic o\&subSeccion=noticia\&id $N=301$

22. Agencia Española de Medicamentos y Productos Sanitarios AEMPS. Seguridad Cadiovascular de los AINE tradicionales: conclusiones de la revisión de los últimos estudios publicados. Madrid: Ministerio de Sanidad SS e I; 2012.

23. Richette P, Latourte A, Frazier A. Safety and efficacy of paracetamol and NSAIDs in osteoarthritis: which drug to recommend? Expert Opin Drug Saf. 2015;14(8):1259-68. DOI: 10.1517/14740338.2015.1056776.

24. OARSI Guidelines | Osteoarthritis Research Society International (OARSI) [Internet]. Disponible en: https://www.oarsi. org/education/oarsi-guidelines

25. Fundació Institut Catalá de Farmacologia. Riscos associats a l'ús concomitant d'opioides i benzodiazepines. Barcelona: Sevei Catalá de la Salut CatSalut; 2017.

26. W. AA. Guía de consenso para el buen uso de analgésicos opioides. Valencia: Sociedad Científica Española de Estudios sobre el Alcohol, el Alcoholismo y las otras Toxicomanías; 2017.

27. Agencia Española de Medicamentos y Productos Sanitarios. Utilización de medicamentos opioides en España durante el periodo 2008-2015. 2017

28. Gouveia N, Rodrigues A, Ramiro S, Eusébio M, Machado PM, Canhao H, et al. The Use of Analgesic and Other PainRelief Drugs to Manage Chronic Low Back Pain: Results from a National Survey. Pain Pract. 2017;17(3):353-65. DOI: 10.1111/papr. 12455

29. Dowell D, Haegerich TM, Chou R. CDC guideline for prescribing opioids for chronic pain-United States, 2016. JAMA. 2016;315(15):1624-45. DOI: 10.1001/jama. 2016.1464

30. Cadogan CA, Ryan C, Cahir C, Bradley CP, Bennett K. Benzodiazepine and Z-drug prescribing in Ireland: analysis of national prescribing trends from 2005 to 2015 . Br J Clin Pharmacol. 2018;84(6):1354-1363. DOI: 10.1111/ bcp. 13570.

31. Petitjean S, Ladewig D, Meier CR, Amrein R, Wiesbeck GA. Benzodiazepine prescribing to the Swiss adult population: results from a national survey of community pharmacies. Int Clin Psychopharmacol. 2007;22(5):292-8. DOI: 10.1097/ YIC.Ob013e328105eOf2.

32. Bachhuber MA, Hennessy S, Cunningham CO, Starrels JL. Increasing Benzodiazepine Prescriptions and Overdose Mortality in the United States, 1996-2013. Am J Public Health. 2016;106(4):686-8. Am J Public Health. 2016;106(4):6868. DOI: 10.2105/AJPH.2016.303061.

33. Haro JM, Palacin C, Vilagut G, Martinez M, Bernal M, Luque I, et al. Prevalencia y factores asociados de los trastornos mentales en España: resultados del estudio ESEMeD-España. Med Clin. 2006;126(12):445-51. 
34. Analgésicos orales de venta sin receta (SPM) para el dolor agudo: un resumen de revisiones Cochrane (Revisión Cochrane traducida). Cochrane Database of Systematic Reviews; 2015.

35. Stosic R, Dunagan F, Palmer H, Fowler T, Adams I. Responsible self-medication: perceived risks and benefits of over-thecounter analgesic use. Int J Pharm Pract. 2011;19(4):23645. DOI: 10.1111/j.2042-7174.2011.00097.x.

36. Ministerio de Sanidad, Consumo y Bienestar Social - Portal Estadístico del SNS - Encuesta Nacional de Salud de España 2017 [Internet]. Disponible en: https://www.mscbs.gob. es/estadEstudios/estadisticas/encuestaNacional/encuesta2017.htm

37. Malo S, Poblador-Plou B, Prados-Torres A, Lallana MJ, Laguna-Berna C, Rabanaque MJ. Poor congruence with guidelines in the use of antibiotics for acute bronchitis: a descriptive study based on electronic health records. Fam Pract. 2016;33(5):471-5. DOI: 10.1093/fampra/ cmw037.

38. Lallana Álvarez MJ, Feja Solana C, Malo Fumanal S, Abad Diez JM BL. Variabilidad de la prescripción de antibióticos en Atención Primaria de los Sectores Sanitarios de Aragón. Rev Esp Salud Pública. 2012;86(6):627-35.

39. Muñoz Giménez N, Mora Corberá E. Tratamientos farmacológicos de la enfemedad de Alzheimer. Estudio comparativo de uso de la Comunidad de Aragón. Farm Prof. 2010;24:60-6.

40. Villar-Fernández I, Bjerrum L, Feja C, Rabanaque MJ. Variability in the Prescription of Cholinesterase Inhibitors and Memantine. Dement Geriatr Cogn Disord. 2009;28(4):3739. DOI: 10.1159/000253845. 